\title{
Modelagem e estimação de parâmetros do processo de produção de etanol em reator batelada por Saccharomyces cerevisiae
}

Modelling and parameter estimation of ethanol production in a batch reactor by Saccharomyces cerevisiae

\author{
C. L. Silva ${ }^{1 *}$; P. L. Santana ${ }^{2}$; C. F. Silva ${ }^{1}$; R. L. Pagano ${ }^{2}$; \\ ${ }_{1}$ Programa de Pós-graduação em Engenharia Química/Laboratório de Bioquímica Industrial/Universidade Federal de \\ Sergipe, 49100-000, São Cristóvão-SE, Brasil \\ ${ }^{2}$ Programa de Pós-graduação em Engenharia Química/Laboratório de Modelagem e Simulação de \\ Processos/Universidade Federal de Sergipe, 49100-000, São Cristóvão-SE, Brasil
}

caroline.aju@gmail.com

(Recebido em 16 de março de 2016; aceito em 12 de abril de 2016)

\begin{abstract}
No presente trabalho foi realizado o estudo cinético da fermentação em batelada com o objetivo de descrever a produção de etanol utilizando a levedura Saccharomyces cerevisiae. A fermentação alcoólica foi realizada em biorreator sob agitação de $175 \mathrm{rpm}$, condição de temperatura igual a $32^{\circ} \mathrm{C} \mathrm{e} \mathrm{pH}$ igual a 3,0 . Com o intuito de descrever o comportamento do crescimento microbiano, da formação de produto e do consumo de substratos foram propostos três modelos matemáticos: um modelo clássico baseado nos balanços de massa para o reator batelada; um modelo baseado na cinética microbiana ponderada pela concentração máxima de microrganismo; e, por último, um modelo proposto na tentativa de prever o comportamento de decaimento celular observado experimentalmente. O modelo que melhor se ajustou aos dados experimentais foi o modelo clássico proposto com base na cinética de Monod. Todos os modelos foram implementados em FORTRAN e resolvidos aplicando a sub-rotina DASSL, por fim, o programa ESTIMA foi utilizado para realizar a estimação dos parâmetros cinéticos de cada modelo, a função objetivo adotada foi a de mínimos quadrados. Os valores estimados dos parâmetros obtidos para o modelo que melhor se ajustou aos dados experimentais foram estatisticamente significativos e fisicamente realistas e descreveram com boa precisão os dados experimentais: $\mu_{\operatorname{máx}}=0,1155 \mathrm{~h}^{-1} ; \mathrm{K}_{1}=0,8984 \mathrm{~h}^{-1} ; \mathrm{K}=0,4738 \mathrm{~h}^{-1}$; $\mathrm{Y}_{\mathrm{X} / \mathrm{S}}=0,0242 ; \mathrm{Y}_{\mathrm{X} / \mathrm{P}}=0,0451$ e $\mathrm{K}_{\mathrm{S}}=24,3852 \mathrm{~g} \cdot \mathrm{L}^{-1}$.
\end{abstract}

Palavras-chave: etanol, batelada, modelo cinético.

In the present study was investigated the kinetic of batch fermentation with the purpose to describe the ethanol production by Saccharomyces cerevisiae. The fermentation was performed in a bioreactor under agitation of $175 \mathrm{rpm}$, temperature condition equal to $32{ }^{\circ} \mathrm{C}$ and $\mathrm{pH}$ at 3.0. In order to describe the behavior of microbial growth, product formation and substrate consumption, three mathematical models were proposed: a classic model based on mass balances for the batch reactor; a model based on microbial kinetics weighted by the maximum concentration of microorganism; and a model proposed in an attempt to predict the cellular decay behavior observed experimentally. The model that best fitted the experimental data was the classic model based on Monod kinetics. All models were implemented in FORTRAN and solved by applying the subroutine DASSL, in the end, the ESTIMA program was used to estimate the kinetic parameters of each model and the objective function adopted was least squares function. The estimated values of the parameters obtained for the best model were statistically significant and physically realistic and describe with good accuracy the experimental data: $\mu_{\max }=0.1155 \mathrm{~h}^{-1} ; \mathrm{K}_{1}=0.8984 \mathrm{~h}^{-1} ; \mathrm{K}=$ $0.4738 \mathrm{~h}-1 ; \mathrm{Y}_{\mathrm{X} / \mathrm{S}}=0.0242 ; \mathrm{Y}_{\mathrm{X} / \mathrm{P}}=0.0451 \mathrm{e} \mathrm{K} \mathrm{S}=24.3852$ g.L $\mathrm{L}^{-1}$.

Keywords: ethanol, batch, kinetic model.

\section{INTRODUÇÃO}

Desde o início deste século, um grande incentivo para o uso de biocombustíveis em substituição à gasolina vem sendo fomentado pelas mais diversas entidades, governamentais ou não. Países como Brasil, Rússia, Estados Unidos, Canadá, Japão Índia, China e países da Europa 
estão interessados em desenvolver mercados internos de biocombustíveis e estabelecer planos para o uso destes biocombustíveis [1].

O etanol pode ser utilizado como um combustível alternativo necessitando de uma modificação relativamente simples nos motores. Apesar da energia equivalente do etanol ser $68 \%$ menor que a dos combustíveis do petróleo, a combustão do etanol é mais limpa e, consequentemente, apresenta uma baixa emissão de substâncias tóxicas [2]. Para a produção de etanol, a levedura Saccharomyces cerevisiae é a espécie mais utilizada, apesar de outros microrganismos serem explorados com este fim, como é o caso a bactéria Zymomonas mobilis [3].

Com o crescente interesse na aplicação industrial da fermentação alcoólica em batelada, vários modelos cinéticos têm sido examinados para o crescimento microbiano, formação de produto e consumo de substrato [4]. Modelos cinéticos para fermentações industriais são normalmente baseados em modelos simples e não estruturados. Além disso, nem todo modelo cinético pode ser diretamente aplicável a uma situação real do processo, portanto o modelo matemático deve partir de um modelo simples que deve ser modificado e adaptado até eventualmente conduzir a um modelo cinético adequado [5].

A determinação de um modelo cinético é considerada a etapa mais importante no estudo teórico-experimental de um processo fermentativo, visto que definirá a taxa de produção e consumo dos principais constituintes, bem como auxiliará no controle do processo, na redução dos custos e no aumento da qualidade do produto. Uma vez que o modelo cinético esteja definido, o mesmo poderá ser utilizado para descrever a produção sob diferentes condições de processo [4].

Neste cenário, o objetivo principal do presente trabalho foi determinar os parâmetros cinéticos que descrevem os dados experimentais da fermentação alcoólica em batelada utilizando a Saccharomyces cerevisiae, propondo um modelo matemático para predição do processo fermentativo, bem como estimar os parâmetros deste modelo utilizando o algoritmo de enxame de partículas implementado no programa ESTIMA.

\section{MATERIAL E MÉTODOS}

\subsection{Composição do meio de cultura}

$\mathrm{O}$ meio de cultura foi composto por fosfato monopotássico $\left(\mathrm{KH}_{2} \mathrm{PO}_{4}\right), 2,5 \mathrm{~g} / \mathrm{L}$; sulfato de magnésio heptahidratado $\left(\mathrm{MgSO}_{4} .7 \mathrm{H}_{2} \mathrm{O}\right), 0,5 \mathrm{~g} / \mathrm{L}$; cloreto de amônio $\left(\mathrm{NH}_{4} \mathrm{Cl}\right), 2,5 \mathrm{~g} / \mathrm{L}$; cloreto de potássio $(\mathrm{KCl}), 0,5 \mathrm{~g} / \mathrm{L}$; extrato de levedura, $3,0 \mathrm{~g} / \mathrm{L}$; sacarose $125 \mathrm{~g} / \mathrm{L}$.

\subsection{Microrganismo}

O microrganismo utilizado para a promover a fermentação alcoólica foi a levedura Saccharomyces cerevisiae proveniente de fermento comercial da marca Lesaffre Saf-instant. A levedura foi adicionada ao meio de cultura a uma concentração de $10 \mathrm{~g} / \mathrm{L}$ [4].

\subsection{Processo fermentativo}

A fermentação foi realizada em um biorreator MS Mistura modelo MSM 205/500 com volume útil de $1 \mathrm{~L}$, sob temperatura de $32{ }^{\circ} \mathrm{C}$ e pH igual a 3,0; com agitação de $175 \mathrm{rpm}$. O processo fermentativo durou $15 \mathrm{~h}$ e foi realizado em triplicata. A cada $3 \mathrm{~h}$ amostras de $25 \mathrm{~mL}$ foram retiradas do reator para realizar acompanhamento das concentrações de biomassa, sacarose, glicose, biomassa e etanol durante o tempo de fermentação.

\subsection{Métodos analíticos}

\subsubsection{Concentração de biomassa}

A concentração de biomassa durante a fermentação foi determinada pelo método descrito por DODIĆ et al. [4] com algumas adaptações. O método utiliza uma dependência espectrofotométrica: absorbância como uma função da concentração de células de levedura.

Antes de iniciar a fermentação foram preparadas suspensões no meio de cultura com leveduras comerciais em diferentes concentrações: 6,$25 ; 12,5 ; 25 ; 50 ; 62,5 \mathrm{~g} / \mathrm{L}$. A suspensão foi feita no 
meio de cultura que foi utilizado no processo fermentativo para cancelar a interferência do meio utilizado durante a medida da absorbância [4].

A concentração real de biomassa foi determinada por meio da medida de peso seco, onde 20 $\mathrm{mL}$ de meio de cultura isento de leveduras e as suspensões preparadas foram colocadas em cadinhos previamente pesado. As amostras foram inicialmente secas em estufa a vácuo a 77,89 $\mathrm{kPa}$ e $38^{\circ} \mathrm{C}$ por $48 \mathrm{~h}$, posteriormente foram congeladas por 25 minutos a $-85{ }^{\circ} \mathrm{C}$ e por fim liofilizadas à temperatura de $-52^{\circ} \mathrm{C}$ por $16 \mathrm{~h}$ até atingirem peso constante.

A absorbância das suspensões foi medida em espectrofotômetro a um comprimento de onda de $660 \mathrm{~nm}$ utilizando o meio de cultura como branco, proporcionando, assim, sua dependência com a concentração de células de levedura:

$\mathrm{A}_{660 \mathrm{~nm}}=3,1318 .[\mathrm{X}]$

sendo $[\mathrm{X}]$ a concentração de biomassa em $\mathrm{g} / \mathrm{L}$. $\mathrm{O}$ coeficiente de determinação $\mathrm{r}^{2}$ desta correlação linear foi igual a 0,9669 .

\subsubsection{Concentração de sacarose e glicose}

A determinação das concentrações de sacarose e glicose foi realizada pelo método DNS. Este método é baseado na capacidade do ácido 3,5-dinitrosalicílico (DNS) ser reduzido pela glicose, açúcar redutor, a um composto nitroamínico análogo e altamente colorido (ácido 3-amino-5nitrosalicílico). No procedimento reacional deste método, uma alíquota de $0,25 \mathrm{~mL}$ da amostra a ser analisada foi transferida para tubo de ensaio e foram adicionados $2 \mathrm{~mL}$ do reagente DNS. A mistura foi agitada vigorosamente e o tubo de ensaio foi colocado em banho-maria com água em ebulição $\left(100{ }^{\circ} \mathrm{C}\right)$ por 5 minutos. A reação foi interrompida imergindo o tubo em banho de água fria e a mistura foi diluída com água destilada. Após homogeneização foi realizada a leitura da intensidade da cor em espectrofotômetro a $480 \mathrm{~nm}$ contra um branco, onde água destilada seguiu o procedimento reacional do método DNS [6,7].

Previamente foram preparadas soluções de glicose com concentrações conhecidas $(10,20,30$, 40 e $50 \mathrm{~g} / \mathrm{L}$ ) para a construção da curva-padrão da concentração de açúcares redutores (AR), de modo a estabelecer a correlação entre a absorbância medida e a quantidade de açúcares redutores presente:

$\mathrm{A}_{480 \mathrm{~nm}}=0,1228 .[\mathrm{AR}]$

sendo [AR] a concentração de açúcares redutores. O coeficiente de determinação $\mathrm{r}^{2}$ desta correlação linear foi igual a 0,9958 .

Para a determinação das concentrações de sacarose e glicose as amostras foram previamente centrifugadas a $4200 \mathrm{rpm}$ por 20 minutos e posteriormente a $12500 \mathrm{rpm}$ por 15 minutos para que a presença da biomassa não interferisse na análise. No caso da determinação do teor de sacarose, que se trata de um açúcar não redutor, as amostras foram submetidas a uma hidrólise ácida antes de serem submetidas ao procedimento reacional do método DNS para possibilitar a determinação do teor de açúcares redutores totais (ART). A concentração de sacarose foi então obtida por meio da subtração da concentração de ART pela concentração de AR [7,8].

\subsubsection{Concentração de etanol}

Foi utilizado um método espectrofotométrico baseado na oxidação do etanol a ácido acético, através da reação com dicromato de potássio, proveniente de uma solução sulfocrômica, em meio ácido. A solução adquire uma tonalidade verde proporcional à concentração de álcool na amostra, possibilitando a leitura da intensidade da cor em espectrofotômetro. Para o procedimento reacional do método uma alíquota $1 \mathrm{~mL}$ da amostra e $1 \mathrm{~mL}$ de solução sulfocrômica foram colocadas em um tubo de ensaio que permaneceu em banho-maria a $30^{\circ} \mathrm{C}$ durante 15 minutos. Após término da reação foram adicionados $8 \mathrm{~mL}$ de água destilada ao tudo de ensaio e a mistura foi homogeneizada. Em seguida foi realizada a leitura da absorbância a um comprimento de onda de $600 \mathrm{~nm}$ contra um branco preparado com água destilada submetida ao mesmo procedimento reacional. A quantificação da concentração de etanol foi feita através da comparação com uma 
curva-padrão construída com concentrações conhecidas de etanol $(0,5 ; 1,0 ; 1,5 ; 2,0$ e $2,5 \%$ (v/v)) [9]:

$\mathrm{A}_{600 \mathrm{~nm}}=0,3189$. [etanol]

o coeficiente de determinação $\mathrm{r}^{2}$ observado para esta correlação linear foi igual a 0,9971 .

Para a determinação da concentração de etanol as amostras também foram centrifugadas a 4200 rpm por 20 minutos e posteriormente a $12500 \mathrm{rpm}$ por 15 minutos para evitar a interferência da presença da biomassa na análise. Além da biomassa outros compostos podem interferir a leitura espectrofotométrica, então foi necessário o preparo de uma amostra desalcoolizada para que fosse possível cancelar o efeito desses interferentes na determinação do teor alcoólico. Para o preparo da amostra desalcoolizada $10 \mathrm{~mL}$ da amostra centrifugada foram colocados sob aquecimento em chapa aquecedora a uma temperatura de aproximadamente $80{ }^{\circ} \mathrm{C}$ para que o etanol presente na amostra evaporasse e ao fim deste processo o volume inicial, de $10 \mathrm{~mL}$ foi recomposto com a adição de água destilada.

A absorbância real da amostra analisada foi dada pela diferença entre a absorbância da amostra centrifugada e a absorbância da amostra desalcoolizada e a concentração de álcool foi determinada por meio da expressão obtida para a curva-padrão.

\subsection{Modelos matemáticos}

Com o objetivo de descrever o comportamento das concentrações de biomassa (X), sacarose $(\mathrm{S})$, glicose $\left(\mathrm{S}_{1}\right)$ e etanol $(\mathrm{P})$ ao longo do processo fermentativo em batelada foram propostos cinco modelos matemáticos. Os modelos propostos foram solucionados através da sub-rotina DASSL [10,11], previamente implementada no compilador FORTRAN.

Algumas hipóteses foram assumidas durante a modelagem matemática: (i) o reator operou sob a hipótese de mistura perfeita, ou seja, as condições foram uniformes ao longo de todo o reator e (ii) a velocidade de agitação de $175 \mathrm{rpm}$ foi suficiente para promover uma adequada transferência de massa e disponibilidade de substrato uniforme.

\subsubsection{Modelo Matemático I (MMI)}

Neste modelo o comportamento de X, S e P durante o processo fermentativo foi proposto com base no balanço de massa para o reator batelada [12].

$\frac{\mathrm{dX}}{\mathrm{dt}}=\mu \cdot \mathrm{X}$

$\frac{\mathrm{dS}}{\mathrm{dt}}=-\frac{\mu \cdot \mathrm{X}}{\mathrm{Y}_{\mathrm{X} / \mathrm{S}}}$

$\frac{\mathrm{dP}}{\mathrm{dt}}=\frac{\mu \cdot \mathrm{X}}{\mathrm{Y}_{\mathrm{X} / \mathrm{P}}}$

sendo $\mathrm{Y}_{\mathrm{X} / \mathrm{S}} \mathrm{o}$ fator de conversão de substrato em biomassa $\left(\mathrm{Y}_{\mathrm{X} / \mathrm{S}}=\mathrm{dX} / \mathrm{dS}\right), \mathrm{Y}_{\mathrm{X} / \mathrm{P}}$ o fator de conversão de substrato em produto $\left(\mathrm{Y}_{\mathrm{X} / \mathrm{P}}=\mathrm{dX} / \mathrm{dP}\right)$ e $\mu\left(\mathrm{h}^{-1}\right)$ a velocidade específica de reprodução do microrganismo, que neste modelo foi definida pela Equação de Monod [13]:

$\mu=\frac{\mu_{\text {máx }} \cdot S}{K_{S}+S}$

sendo $\mu_{\text {máx }}\left(h^{-1}\right)$ a máxima velocidade específica de crescimento e $K_{S}(g / L)$ a constante de saturação.

Para descrever o comportamento de $S_{1}$ foi desenvolvido um modelo baseado na taxa de reação para a glicose no sistema de reações da fermentação alcoólica:

$\mathrm{C}_{12} \mathrm{H}_{22} \mathrm{O}_{11}$ (sacarose) $\stackrel{\mathrm{H}_{2} \mathrm{O}}{\longrightarrow} \mathrm{C}_{6} \mathrm{H}_{12} \mathrm{O}_{6}$ (glicose) $+\mathrm{C}_{6} \mathrm{H}_{12} \mathrm{O}_{6}$ (frutose) 
$\mathrm{C}_{6} \mathrm{H}_{12} \mathrm{O}_{6}$ (glicose $) \rightarrow 2 \mathrm{C}_{2} \mathrm{H}_{5} \mathrm{OH}($ etanol $)+2 \mathrm{CO}_{2}$

Sob a hipótese de que estas reações são elementares, tem-se:

$r_{\text {glicose }}=\frac{\mathrm{dS}_{1}}{\mathrm{dt}}=\mathrm{K}_{1} \cdot \mathrm{S}-\mathrm{K} \cdot \mathrm{S}_{1}$

sendo $\mathrm{K}_{1}$ e $\mathrm{K}\left(\mathrm{h}^{-1}\right)$ as constantes cinéticas das reações (8) e (9), respectivamente.

\subsubsection{Modelo Matemático II (MMII)}

Neste modelo foi proposta uma modificação com relação ao modelo MMI. As equações que modelam o comportamento de X, S ,S ${ }_{1}$ e P (Equações (4), (5), (6) e (10), respectivamente) foram mantidas, mas equação de Monod utilizada para determinar a velocidade específica de crescimento celular foi substituída por equação proposta por DODIĆ et al. [4] para a modelagem matemática de fermentação alcoólica a partir de suco de beterraba:

$\mu=\mu_{\text {máx }}\left(1-\frac{x}{x_{\text {máx }}}\right)$

sendo $\mathrm{X}_{\text {máx }}$ a máxima concentração celular atingida, obtida experimentalmente.

\subsubsection{Modelo Matemático III (MMIII)}

Este terceiro modelo foi proposto com o intuito de descrever o decaimento celular ao final da fermentação. Neste caso, foi incorporado à equação de comportamento celular ao longo do tempo de fermentação um termo definido como taxa específica de morte celular, $\mathrm{K}_{\mathrm{d}}\left(\mathrm{g}^{2} \cdot \mathrm{L}^{-2} \cdot \mathrm{h}^{-1}\right)$ :

$\frac{\mathrm{dX}}{\mathrm{dt}}=\left(\mu-K_{d}\right) \mathrm{X}$

As Equações (5), (6), (10) e (11) apresentadas nos modelos MMI e MMII foram utilizadas neste modelo.

\subsection{Método de estimação de parâmetros}

O ajuste dos parâmetros dos modelos cinéticos propostos foi realizado através da aplicação do programa ESTIMA, que é baseado no algoritmo heurístico de enxame de partículas, PSO (Particle Swarm Optimization) [14]. A média dos valores dos resultados experimentais em triplicata foram utilizados para o cálculo dos parâmetros e ajuste aos modelos propostos a função objetivo adotada foi a função de mínimos quadrados Equação (13). As regiões de confiança dos parâmetros estimados foram obtidas considerando a metodologia apresentada por Enzweiler et al. [15].

$\mathrm{F}_{\mathrm{obj}}=\sum_{\mathrm{i}=1}^{\mathrm{NE}}\left(\mathrm{y}_{\mathrm{i}}^{\mathrm{e}}-\mathrm{y}_{\mathrm{i}}^{\mathrm{m}}\right)^{2}$

As equações dos modelos, Equações (4) a (7) e (10) a (12), foram adimensionadas a partir das Equações (14) a (18) e estão apresentadas nas Tabelas 1 a 3. O adimensionamento das equações que descrevem o modelo é uma ferramenta que permite uma melhor compreensão das variáveis significativas do problema [16].

$$
\begin{aligned}
& \mathrm{X}^{*}=\frac{\mathrm{X}-\mathrm{X}_{0}}{\mathrm{X}_{\mathrm{F}}-\mathrm{X}_{0}} \\
& \mathrm{~S}^{*}=\frac{\mathrm{S}-\mathrm{S}_{0}}{\mathrm{~S}_{\mathrm{F}}-\mathrm{S}_{0}}
\end{aligned}
$$




$$
\begin{aligned}
& \mathrm{S}_{1}{ }^{*}=\frac{\mathrm{S}_{1}-\mathrm{S}_{10}}{\mathrm{~S}_{1 \mathrm{~F}}-\mathrm{S}_{10}} \\
& \mathrm{P}^{*}=\frac{\mathrm{P}}{\mathrm{P}_{\mathrm{F}}} \\
& \mathrm{t}^{*}=\frac{\mathrm{t}}{\mathrm{t}_{\mathrm{F}}}
\end{aligned}
$$

As constantes presentes nas equações estão apresentadas na Tabela 4 e os parâmetros a serem estimados em cada um dos modelos propostos estão listados nas Tabela 5.

Tabela 1: Equações adimensionais do MMI

\begin{tabular}{ll}
\hline Biomassa & $\frac{\mathrm{dX}^{*}}{\mathrm{dt}^{*}}=\mu^{*}\left(\gamma_{X}+\mathrm{X}^{*}\right)$ \\
Sacarose & $\frac{\mathrm{dS}^{*}}{\mathrm{dt}^{*}}=-\frac{\mu^{*}\left(\lambda_{S 1}+\lambda_{S 2} \cdot \mathrm{X}^{*}\right)}{\mathrm{Y}_{\mathrm{X} / \mathrm{S}}}$ \\
Glicose & $\frac{\mathrm{d} S_{1}^{*}}{\mathrm{dt}^{*}}=K_{1}^{*}\left(\lambda_{S 10}+\lambda_{S 11} \cdot \mathrm{S}^{*}\right)-\mathrm{K}^{*}\left(\gamma_{S 1}+S_{1}^{*}\right)$ \\
Etanol & $\frac{\mathrm{dP}^{*}}{\mathrm{dt}^{*}}=\frac{\mu^{*}\left(\lambda_{P 1}+\lambda_{P 2} \cdot \mathrm{X}^{*}\right)}{\mathrm{Y}_{\mathrm{X} / \mathrm{P}}}$ \\
Cinética & $\mu^{*}=\frac{\mu_{m a x}^{*}\left(\gamma_{S}+\mathrm{S}^{*}\right)}{K_{S}^{*}+\gamma_{S}+\mathrm{S}^{*}}$
\end{tabular}

Tabela 2: Equações adimensionais do MMII

\begin{tabular}{ll}
\hline Biomassa & $\frac{\mathrm{dX}^{*}}{\mathrm{dt}^{*}}=\mu^{*}\left(\gamma_{x}+\mathrm{X}^{*}\right)$ \\
Sacarose & $\frac{\mathrm{dS}^{*}}{\mathrm{dt}^{*}}=-\frac{\mu^{*}\left(\lambda_{S 1}+\lambda_{S 2} \cdot \mathrm{X}^{*}\right)}{\mathrm{Y}_{\mathrm{X} / \mathrm{S}}}$ \\
Glicose & $\frac{\mathrm{d} S_{1}^{*}}{\mathrm{dt}^{*}}=K_{1}^{*}\left(\lambda_{S 10}+\lambda_{S 11} \cdot \mathrm{S}^{*}\right)-\mathrm{K}^{*}\left(\gamma_{S 1}+S_{1}^{*}\right)$ \\
Etanol & $\frac{\mathrm{dP}^{*}}{\mathrm{dt}^{*}}=\frac{\mu^{*}\left(\lambda_{P 1}+\lambda_{P 2} \cdot \mathrm{X}^{*}\right)}{\mathrm{Y}_{\mathrm{X} / \mathrm{P}}}$ \\
Cinética & $\mu^{*}=\mu_{\max }^{*}\left(1-\frac{\mathrm{X}_{0}}{\mathrm{X}_{\operatorname{máx}}}-\frac{\left(X_{F}-X_{0}\right) \cdot X^{*}}{X_{\max }}\right)$
\end{tabular}

Tabela 3: Equações adimensionais do MMIII

\begin{tabular}{lc}
\hline Biomassa & $\frac{\mathrm{dX}^{*}}{\mathrm{dt}^{*}}=\left(\mu^{*}-K_{d}^{*}\right)\left(\gamma_{x}+\mathrm{X}^{*}\right)$ \\
Sacarose & $\frac{\mathrm{dS}^{*}}{\mathrm{dt}^{*}}=-\frac{\mu^{*}\left(\lambda_{S 1}+\lambda_{S 2} \cdot \mathrm{X}^{*}\right)}{\mathrm{Y}_{\mathrm{X} / \mathrm{S}}}$ \\
Glicose & $\frac{\mathrm{d} S_{1}^{*}}{\mathrm{dt}^{*}}=K_{1}^{*}\left(\lambda_{S 10}+\lambda_{S 11} \cdot \mathrm{S}^{*}\right)-\mathrm{K}^{*}\left(\gamma_{S 1}+S_{1}^{*}\right)$ \\
Etanol & $\frac{\mathrm{dP}^{*}}{\mathrm{dt}^{*}}=\frac{\mu^{*}\left(\lambda_{P 1}+\lambda_{P 2} \cdot \mathrm{X}^{*}\right)}{\mathrm{Y}_{\mathrm{X} / \mathrm{P}}}$ \\
Cinética & $\mu^{*}=\mu_{\max }^{*}\left(1-\frac{\mathrm{X}_{0}}{\mathrm{X}_{\operatorname{máx}}}-\frac{\left(X_{F}-X_{0}\right) \cdot X^{*}}{X_{\max }}\right)$
\end{tabular}


Tabela 4: Constantes das equações adimensionais dos modelos MMI, MMII, MMIII

\begin{tabular}{ccc}
\hline$\gamma_{x}=\frac{X_{0}}{X_{F}-X_{0}}$ & $\lambda_{S 10}=\frac{S_{0}}{S_{1 F}-S_{10}}$ & $\mu_{\text {max }}^{*}=\mu_{\max } \cdot t_{F}$ \\
$\lambda_{S 1}=\frac{X_{0}}{S_{F}-S_{0}}$ & $\lambda_{S 11}=\frac{S_{F}-S_{0}}{S_{1 F}-S_{10}}$ & $K_{S}^{*}=\frac{K_{S}}{S_{F}-S_{0}}$ \\
$\lambda_{S 2}=\frac{X_{F}-X_{0}}{S_{F}-S_{0}}$ & $\gamma_{S 1}=\frac{S_{10}}{S_{1 F}-S_{10}}$ & $\gamma_{S}=\frac{S_{0}}{S_{F}-S_{0}}$ \\
$K_{1}^{*}=K_{1} \cdot t_{F}$ & $\lambda_{P 1}=\frac{X_{0}}{P_{F}}$ & $K_{d}^{*}=K_{d} \cdot t_{F}$ \\
$K^{*}=K \cdot t_{F}$ & $\lambda_{P 2}=\frac{X_{F}-X_{0}}{P_{F}}$ & \\
\hline
\end{tabular}

Tabela 5 - Parâmetros a serem ajustados

\begin{tabular}{ccc}
\hline Modelo & Número de parâmetros & Parâmetros a serem estimados \\
\hline MMI & 6 & $\mu_{\text {max }}^{*}, K_{1}^{*}, K^{*}, \mathrm{Y}_{\mathrm{X} / \mathrm{S}}, \mathrm{Y}_{\mathrm{X} / \mathrm{P}}, K_{S}^{*}$ \\
MMII & 5 & $\mu_{\text {max }}^{*}, K_{1}^{*}, K^{*}, \mathrm{Y}_{\mathrm{X} / \mathrm{S}}, \mathrm{Y}_{\mathrm{X} / \mathrm{P}}$ \\
MMIII & 6 & $\mu_{\text {max }}^{*}, K_{1}^{*}, K^{*}, \mathrm{Y}_{\mathrm{X} / \mathrm{S}}, \mathrm{Y}_{\mathrm{X} / \mathrm{P}}, K_{d}^{*}$ \\
\hline
\end{tabular}

\section{RESULTADOS E DISCUSSÃO}

O processo fermentativo descontínuo para a produção de etanol durou $15 \mathrm{~h}$. A média e o desvio padrão das medidas das concentrações de biomassa [X], sacarose [S], glicose [S1] e etanol [P] estão apresentados graficamente em função do tempo de fermentação na Figura 1.
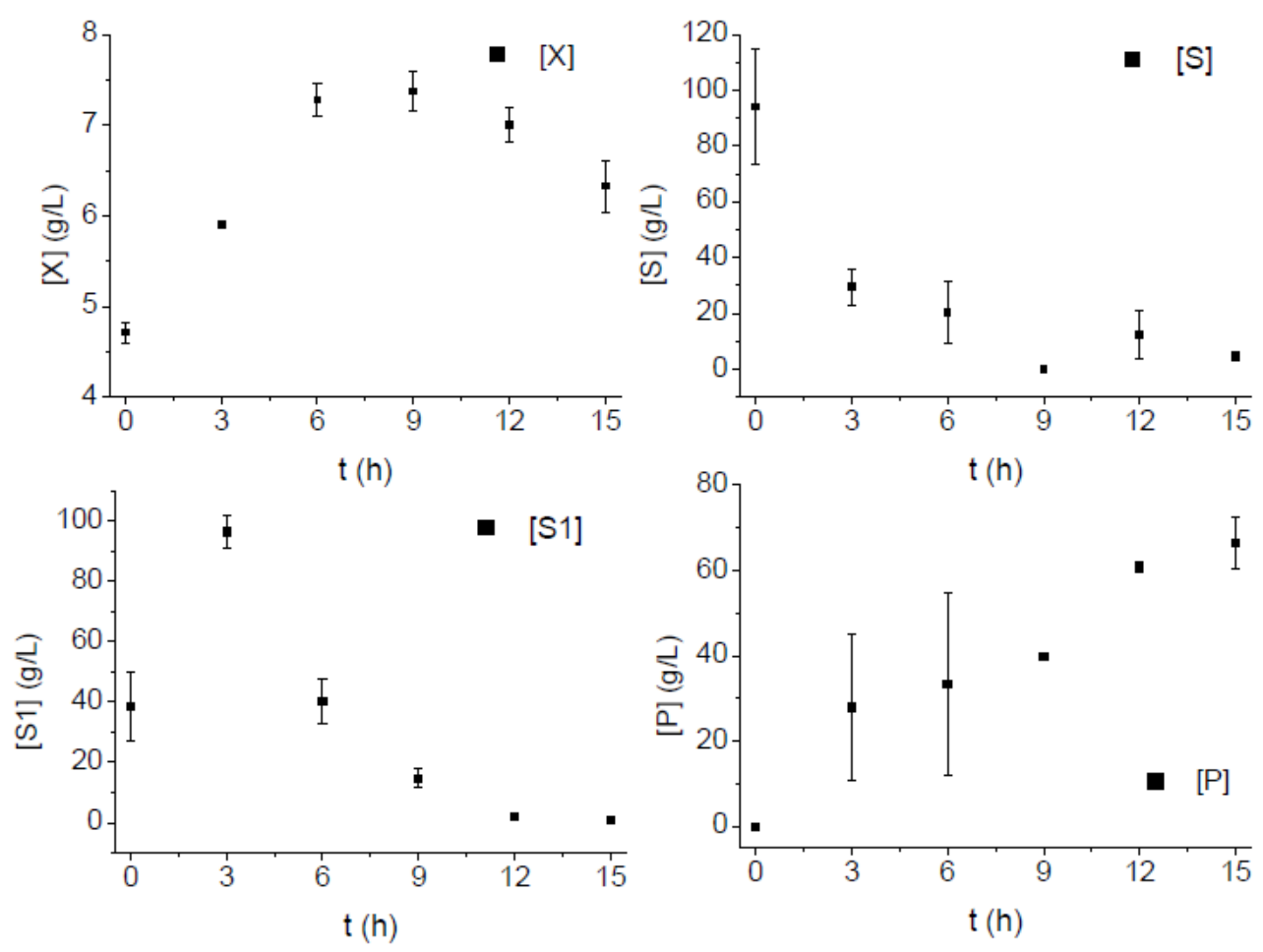

Figura 1: Resultados experimentais do processo descontínuo: médias e desvios padrões 
Analisando os gráficos apresentados na Figura 1, é possível notar que a concentração de biomassa atingiu seu máximo depois de $9 \mathrm{~h}$ de fermentação $\left(\mathrm{X}_{\operatorname{máx}}=7,375 \mathrm{~g} / \mathrm{L}\right)$, período no qual também se observa o mínimo valor da concentração de sacarose, isto se deve ao fato de a levedura estar metabolizando a sacarose tanto para o crescimento celular, quanto para a formação da glicose. A partir das $9 \mathrm{~h}$ de fermentação a concentração de biomassa diminui, indicando a fase de morte celular, neste período além da baixa concentração de substrato, há uma grande concentração de etanol que pode estar agindo como inibidor no crescimento celular. Observando o gráfico apresentado para a glicose, sua formação ocorreu nas primeiras $3 \mathrm{~h}$ através do consumo da sacarose e, a partir deste momento, há seu consumo para a formação de etanol. Deste modo, é possível perceber que o comportamento das concentrações de biomassa, sacarose e etanol apresentados nos gráficos da Figura 1 se mostram condizentes com o comportamento previsto pela literatura clássica sobre processos fermentativos.

Com base nos dados experimentais e nas equações adimensionais propostas nos modelos, os valores dos parâmetros foram obtidos através do ESTIMA e estão apresentados na Tabela 6, bem como os limites inferiores e superiores da região de confiança da função objetivo linearizada. Os valores das respectivas funções objetivos estão apresentados na Tabela 7.

Tabela 6: Valores adimensionais dos parâmetros e limites de confiança da função objetivo linearizada

\begin{tabular}{ccccc}
\hline Modelo & Parâmetros & Valor estimado & Limite inferior & Limite superior \\
\hline \multirow{6}{*}{ MMI } & $\mu_{\text {max }}^{*}$ & 1,73181 & $-13,4533$ & 16,9169 \\
& $K_{1}^{*}$ & 13,4756 & $-43,0195$ & 69,9707 \\
& $K^{*}$ & 7,10664 & $-25,7917$ & 40,005 \\
& $\mathrm{Y}_{\mathrm{X} / \mathrm{S}}$ & 0,0242 & 0,00514 & 0,04326 \\
& $\mathrm{Y}_{\mathrm{X} / \mathrm{P}}$ & 0,04509 & $-0,02348$ & 0,11365 \\
& $K_{S}^{*}$ & $-0,27168$ & $-7,81868$ & 7,27532 \\
\hline \multirow{4}{*}{ MMII } & $\mu_{\max }^{*}$ & 5,1164 & $-4,06185$ & 14,2946 \\
& $K_{1}^{*}$ & 37,5556 & $-653,156$ & 728,267 \\
& $K^{*}$ & 20,5343 & $-326,089$ & 367,158 \\
& $\mathrm{Y}_{\mathrm{X} / \mathrm{S}}$ & 0,02795 & 0,01768 & 0,03822 \\
& $\mathrm{Y}_{\mathrm{X} / \mathrm{P}}$ & 0,04872 & $-0,01854$ & 0,11597 \\
\hline \multirow{4}{*}{ MMIII } & $\mu_{\max }^{*}$ & 6,02782 & $-9,88614$ & 21,9418 \\
& $K_{1}^{*}$ & 50,3487 & $-3105,17$ & 3205,87 \\
& $K^{*}$ & 27,2114 & $-1497,92$ & 1552,34 \\
& $K^{*}{ }^{*} / \mathrm{S}$ & 0,03235 & $-0,02016$ & 0,08485 \\
& $\mathrm{Y}_{\mathrm{X} / \mathrm{P}}$ & 0,05591 & $-0,05607$ & 0,16788 \\
& $K_{d}^{*}$ & 0,09018 & $-0,8504$ & 1,03076 \\
\hline
\end{tabular}

Tabela 7: Valores da função objetivo

\begin{tabular}{cc}
\hline Modelo & Função Objetivo \\
\hline MMI & 0,5291 \\
MMII & 0,6879 \\
MMIII & 0,6334 \\
\hline
\end{tabular}

Com base nos resultados apresentados na Tabela 6 foi possível concluir que o MMI foi o que melhor se ajustou aos dados experimentais por apresentar o menor valor de função objetivo dentre os modelos testados. Através dos dados expressos na Tabela 6, é possível perceber que vários dos parâmetros calculados não são significativos estatisticamente dentro da região de confiança definida pela função objetivo linearizada. Então se fez necessária uma análise dos limites de confiança definidos pelo enxame de partículas considerando $95 \%$ confiança e as 
Figuras 2 a 4 apresentam as regiões de confiança construídas para cada um dos modelos propostos.
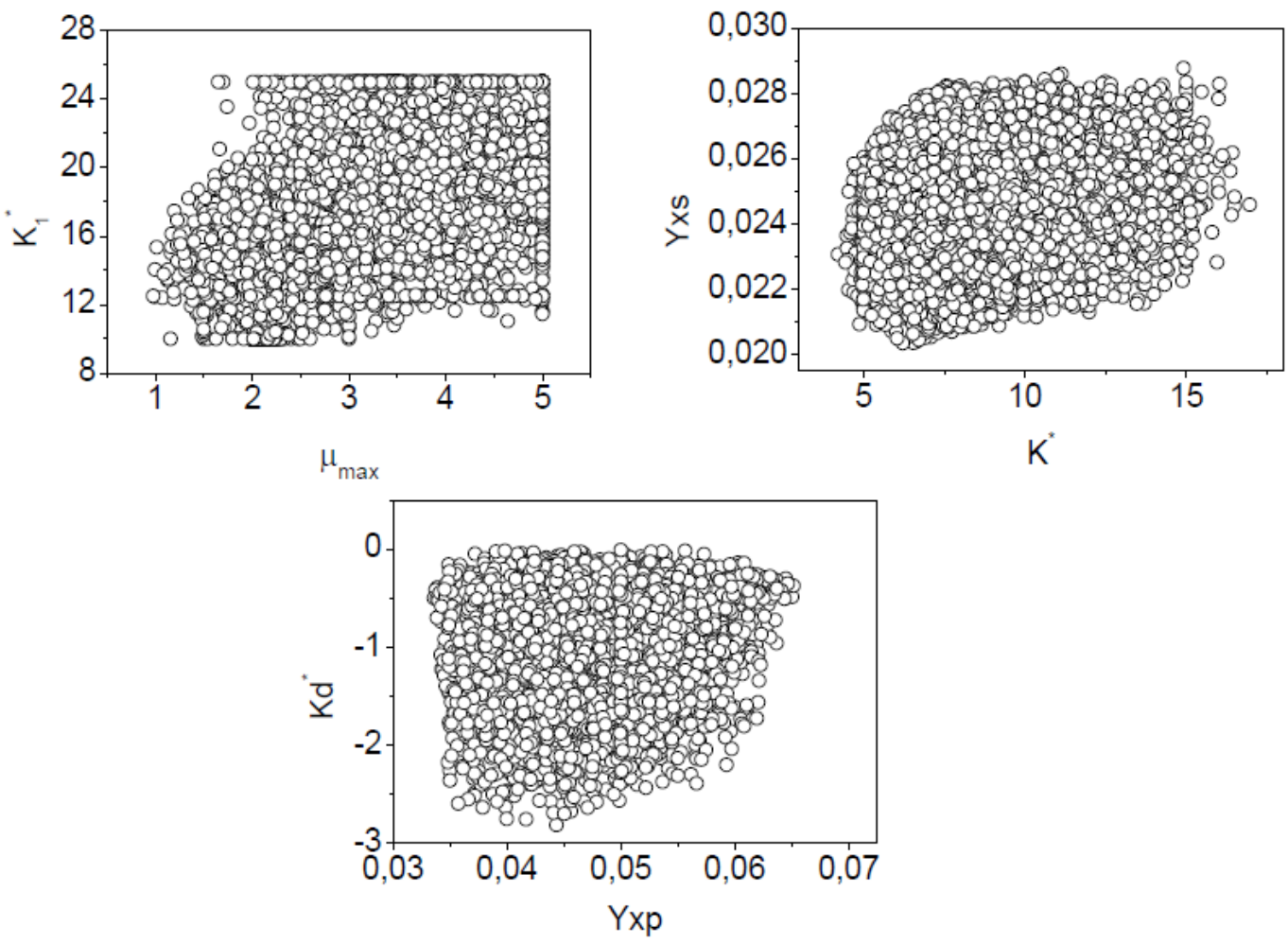

Figura 2: Região de confiança do MMI
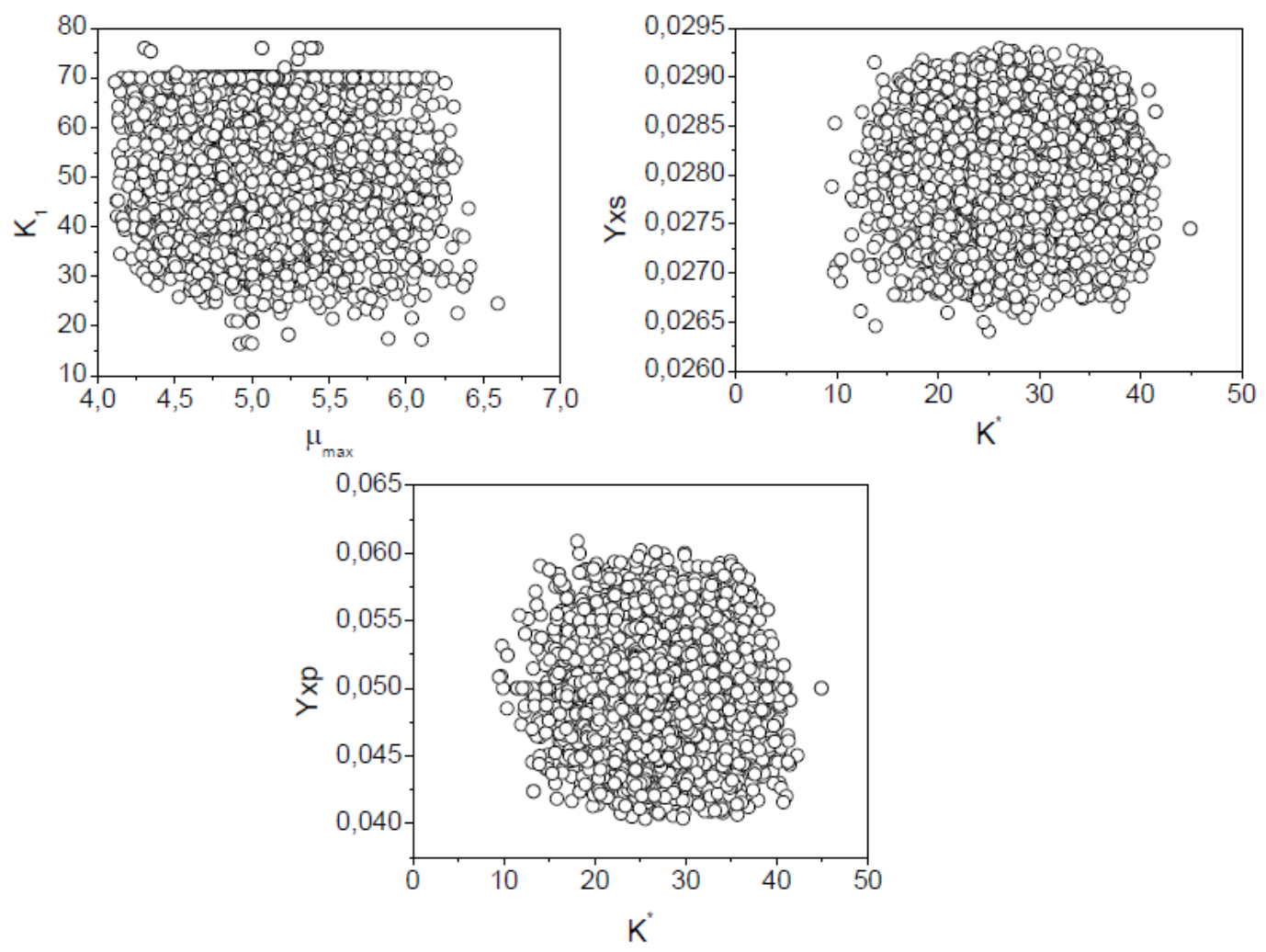

Figura 3: Região de confiança do MMII 


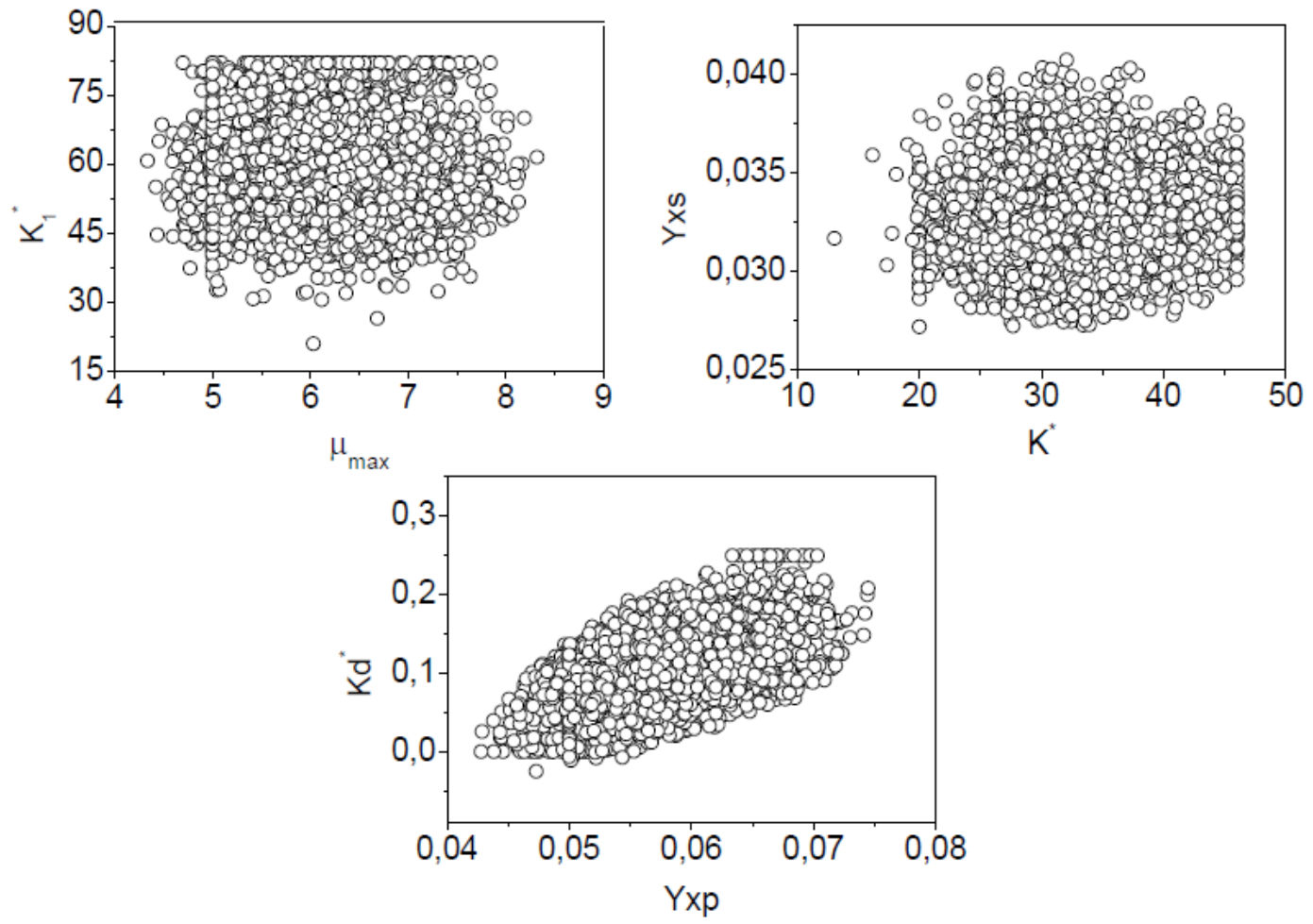

Figura 4: Região de confiança do MMIII

As regiões de confiança, em uma situação ideal, deveriam apresentar um formato elíptico, sendo que o centro geométrico representaria o valor dos parâmetros estimados e suas extremidades os limites de confiança de cada parâmetro. Outro detalhe é fato que essas regiões seriam simétricas e deveriam possuir eixos paralelos aos eixos coordenados, ou seja, não apresentariam nenhum tipo de rotação, como o visualizado na Figura 4 no gráfico de $\mathrm{K}_{\mathrm{d}}$ versus $Y_{\mathrm{XP}}$. Os fenômenos que provocam a distorção da região de confiança são atribuídos à existência de correlações paramétricas, ou seja, a alteração do valor em um parâmetro (aumento ou diminuição) provoca uma alteração nos demais parâmetros. Por fim, deve-se constatar que o cálculo do limite de confiança sem considerar as correlações existentes pode levar a resultados nos quais parâmetros estimados podem ser considerados não-significativos estatisticamente, influenciando diretamente na rejeição de modelos cinéticos precocemente.

A alta correlação entre os parâmetro impossibilitou a definição de todas as fronteiras das regiões de confiança apresentadas nas Figuras 2 a 4, contudo é possível comprovar que os parâmetros são significativos estatisticamente, pois o zero não faz parte do intervalo de confiança. Exceto para o caso do parâmetro $\mathrm{K}_{\mathrm{d}}{ }^{*}$ (MMIII) que não é estatisticamente significativo, pois ao observar a Figura 4 é possível perceber que o zero faz parte da sua região de confiança.

A Tabela 8 apresenta os valores dos parâmetros, com sua respectiva dimensão, estimados para cada um dos modelos em estudo. E nas Figuras 5 a 9 estão apresentados os gráficos comparativos entre os dados obtidos experimentalmente e os dados preditos pelos modelos. Observando esses gráficos, nota-se que nenhum dos modelos testados foi capaz de prever de forma satisfatória os comportamentos de morte celular das leveduras e formação de produto. 
Tabela 8: Valores dos parâmetros dos modelos com dimensão

\begin{tabular}{ccc}
\hline Modelo & Parâmetros & Valor estimado \\
\hline & $\mu_{\text {máx }}\left(\mathrm{h}^{-1}\right)$ & 0,1155 \\
& $\mathrm{~K}_{1}\left(\mathrm{~h}^{-1}\right)$ & 0,8984 \\
MMI & $\mathrm{K}\left(\mathrm{h}^{-1}\right)$ & 0,4738 \\
& $\mathrm{Y}_{\mathrm{X} / \mathrm{S}}$ & 0,0242 \\
& $\mathrm{Y}_{\mathrm{X} / \mathrm{P}}$ & 0,0451 \\
& $\mathrm{~K}_{\mathrm{S}}\left(\mathrm{g} . \mathrm{L}^{-1}\right)$ & 24,3752 \\
\hline & $\mu_{\text {máx }}\left(\mathrm{h}^{-1}\right)$ & 0,3411 \\
MMII & $\mathrm{K}_{1}\left(\mathrm{~h}^{-1}\right)$ & 2,5037 \\
& $\mathrm{~K}\left(\mathrm{~h}^{-1}\right)$ & 1,3690 \\
& $\mathrm{Y}_{\mathrm{X} / \mathrm{S}}$ & 0,0279 \\
& $\mathrm{Y}_{\mathrm{X} / \mathrm{P}}$ & 0,0487 \\
\hline \multirow{4}{*}{ MMIII } & $\mu_{\text {máx }}\left(\mathrm{h}^{-1}\right)$ & 0,4019 \\
& $\mathrm{~K}_{1}\left(\mathrm{~h}^{-1}\right)$ & 3,3566 \\
& $\mathrm{~K}\left(\mathrm{~h}^{-1}\right)$ & 1,8141 \\
& $\mathrm{Y}_{\mathrm{X} / \mathrm{S}}$ & 0,0323 \\
& $\mathrm{Y}_{\mathrm{X} / \mathrm{P}}$ & 0,0559 \\
& $\mathrm{Kd}\left(\mathrm{h}^{-1}\right)$ & 0,0060 \\
\hline
\end{tabular}
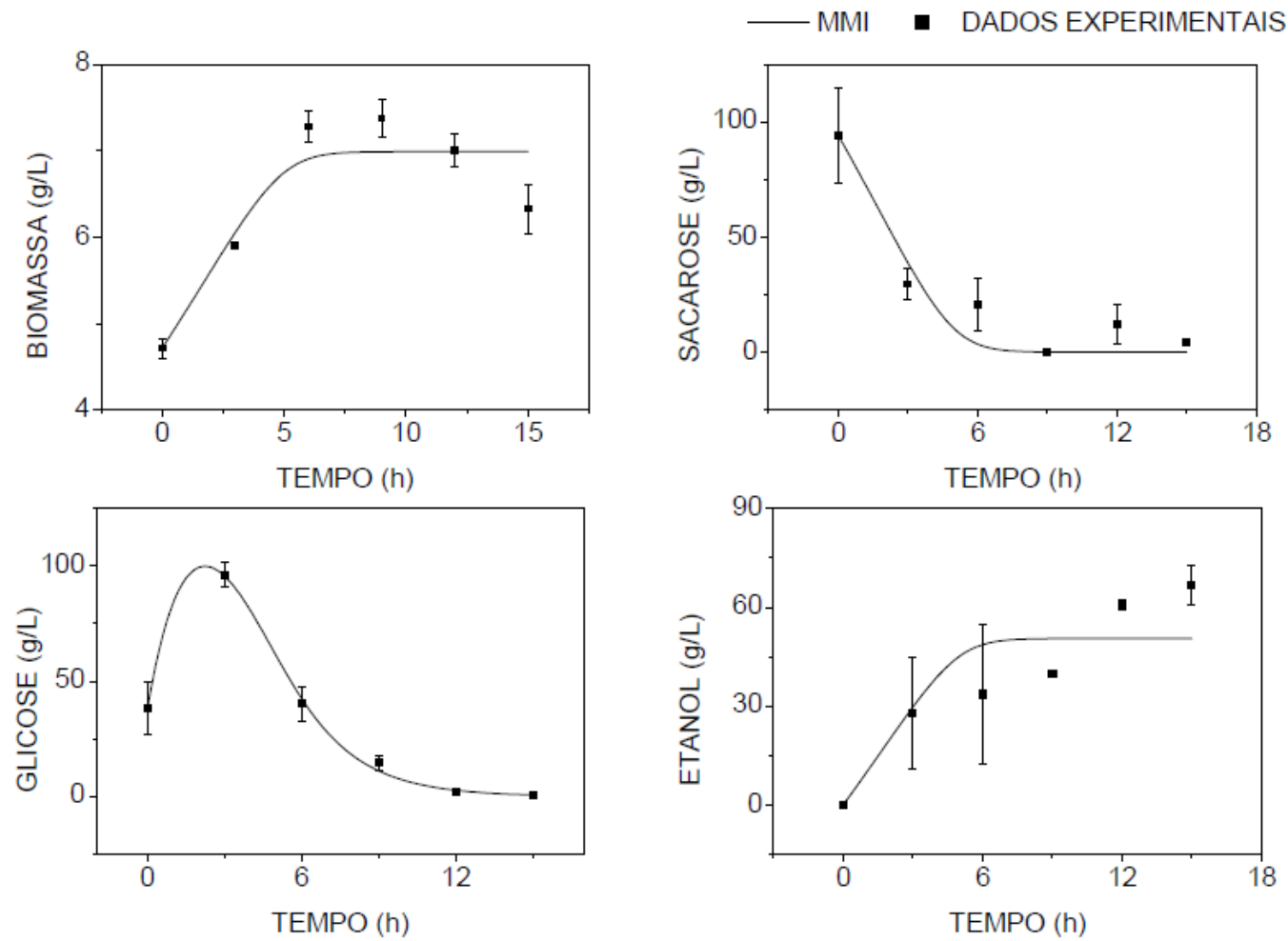

Figura 5: MMI: valores experimentais $x$ valores preditos pelo modelo 

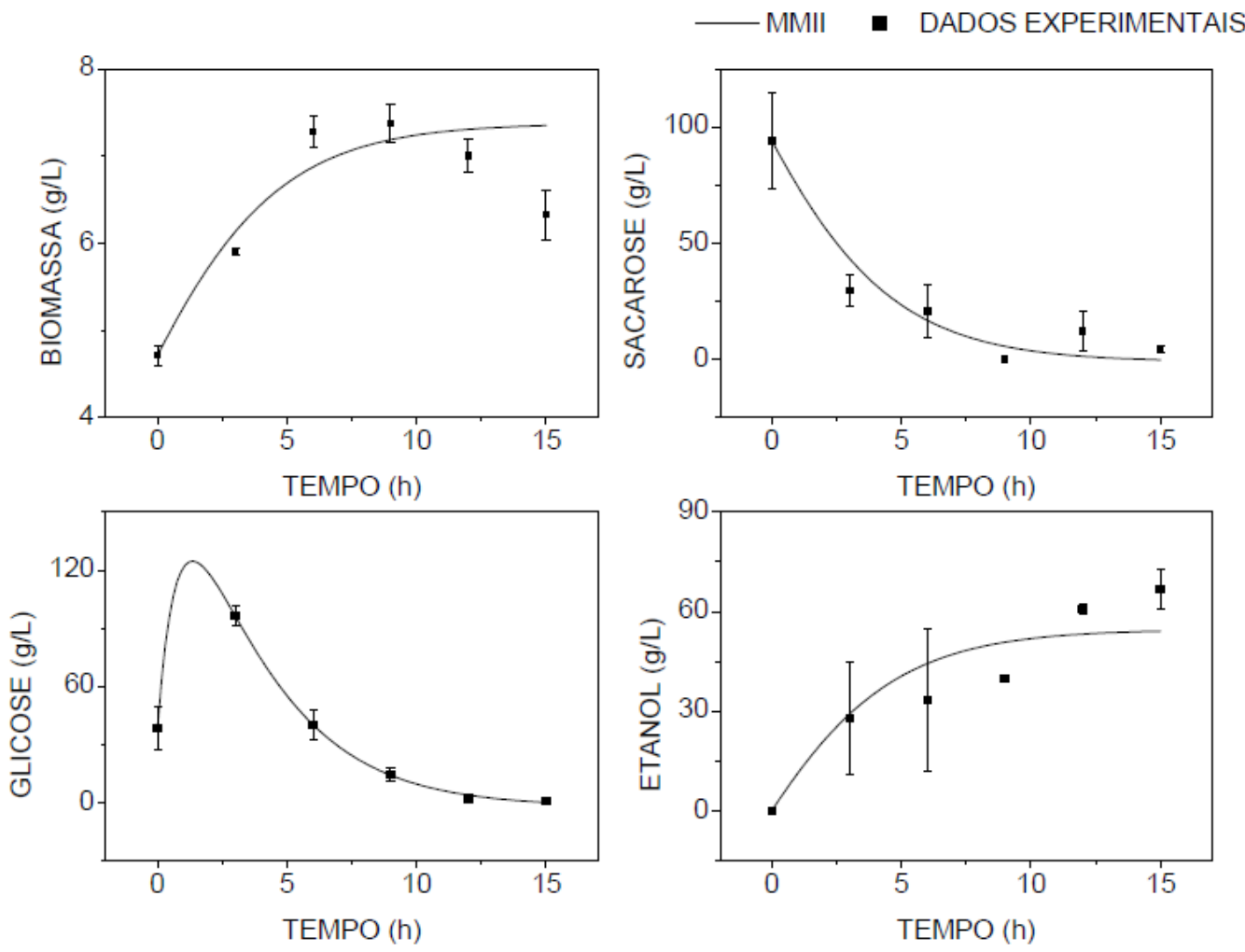

Figura 6: MMII: valores experimentais $x$ valores preditos pelo modelo
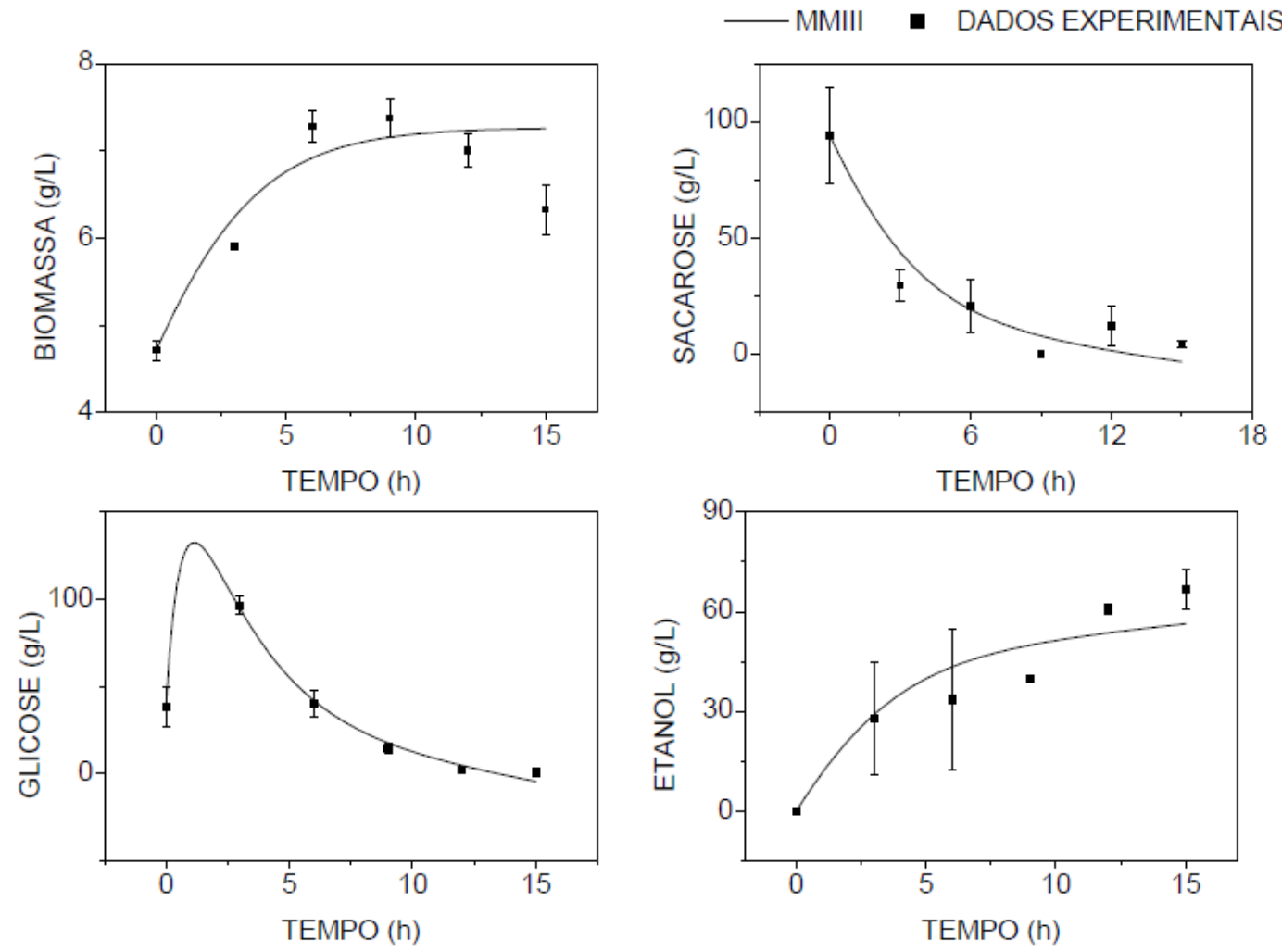

Figura 7: MMIII: valores experimentais $x$ valores preditos pelo modelo 


\section{CONCLUSÃO}

Considerando o exposto neste trabalho, foi possível concluir que os resultados experimentais obtidos estão coerentes com o descrito pela literatura clássica. Na segunda parte deste trabalho, três modelos matemáticos para a cinética de fermentação foram estudados e avaliados para descrever o processo de produção de etanol em batelada por Saccharomyces cerevisiae. Com base nos resultados apresentados foi possível concluir que o modelo matemático I (MMI) foi o que melhor se ajustou aos dados experimentais, contudo, diferentes modelos podem ser testados, bem como a utilização de uma análise estatística mais detalhada pode ser empregada para descriminar estes modelos em uma continuação deste trabalho.

O MMI possui seis parâmetros que foram ajustados aos dados experimentais por meio do programa ESTIMA, o qual utiliza o algoritmo de enxame de partículas. Os valores estimados foram: $\mu_{\text {máx }}=0,1155 \mathrm{~h}^{-1} ; \mathrm{K}_{1}=0,8984 \mathrm{~h}^{-1} ; \mathrm{K}=0,4738 \mathrm{~h}^{-1} ; \mathrm{Y}_{\mathrm{X} / \mathrm{S}}=0,0242 ; \mathrm{Y}_{\mathrm{X} / \mathrm{P}}=0,0451 \mathrm{e} \mathrm{K}_{\mathrm{S}}=$ 24,3852 g.L $\mathrm{L}^{-1}$; foram significativos e fisicamente realísticos e o menor valor encontrado para a função objetivo foi igual a 0,5291 .

\section{AGRADECIMENTOS}

Os autores gostariam de agradecer ao CNPq (Projeto UNIVERSAL - Processo 484493/20124), FATIPEC/SE (Projeto PRONEM - EDITAL FAPITEC/SE/FUNTEC/CNPq N ${ }^{\circ}$ 10/2011 e Projeto PPP - EDITAL FAPITEC / SE / FUNTEC / CNPq N 04/2011) e ao PEQ/UFS pelo suporte financeiro e colaboração.

\section{REFERÊNCIAS BIBLIOGRÁFICAS}

1. Mussatto SI, Dragone G, Guimarães PMR, Silva JPA, Carneiro LM, Roberto IC, et al. Technological trends, global market, and challenges of bio-ethanol production. Biotechnol Adv 2010;28(6):817-30, doi:10.1016/j.biotechadv.2010.07.001.

2. Krylova AY, Kozyukov EA, Lapidus AL. Ethanol and Diesel Fuel from Plant Raw Materials: A Review. Solid Fuel Chem 2008;42(6):358-64, doi:10.3103/S0361521908060062.

3. Bai FW, Anderson WA, Moo-Young M. Ethanol fermentation technologies from sugar and starch feedstocks. Biotechnol Adv 2008;26(1):89-105, doi:10.1016/j.biotechadv.2007.09.002.

4. Dodić JM, Vučurović DG, Dodić SN, Grahovac JA, Popov SD, Nedeljković NM. Kinetic modelling of batch ethanol production from sugar beet raw juice. Appl Energy. 2012;99:192-7, doi:10.1016/j.apenergy.2012.05.016.

5. Birol G, Doruker P, Kirdar B, Önsan ZI, Ülgen K. Mathematical description of ethanol fermentation by immobilised Saccharomyces cerevisiae. Process Biochem. 1998;33(7):763-71.

6. Gonçalves C, Rodriguez-Jasso RM, Gomes N, Teixeira J a., Belo I. Adaptation of dinitrosalicylic acid method to microtiter plates. Anal Methods. 2010;2(12):2046, doi:10.1039/c0ay00525h.

7. Rodrigues DCGA, Santos TT. Manual de Práticas de Processos Bioquímicos. Universidade do Estado do Rio de Janeiro. 2011.

8. Vasconcelos NM, Pinto GAS, Aragão FAS. Determinação de Açúcares Redutores pelo Ácido 3,5Dinitrosalicílico: Histórico do Desenvolvimento do Método e Estabelecimento de um Protocolo parao Laboratório de Bioprocessos. Boletim de Pesquisa 88. 2007;1-22.

9. Magrí AD, Magrí AL, Balestrieri F, Sacchini A, Marini D. Spectrophotometric micro-method for the determination of ethanol in commercial beverages. Fresenius J Anal Chem 1997;357:985-8.

10. Dassl Code (Differential Algebraic System Solver) [computer program]. Lawrence Livermore National Laboratory, Livermore: 1989.

11. Vieira RC, Biscaia Jr EC. Direct methods for consistent initialization of DAE systems. Comput. \& Chem. Eng. 2001 Sep 15;25(9-10):1299-311.

12. Fogler HS. Elementos de Engenharia das Reações Químicas. 4th ed. Rio de Janeiro: LTC; 2009. $853 \mathrm{p}$.

13. Hiss H. Cinética de Processos Fermentativos. In: Schmidell, W; Lima, U; Aquarone, E; Borzani W, editor. Biotecnologia Industrial: Engenharia Bioquímica. São Paulo: Edgard Blücher; 2001. p. 93122. 
14. Noronha FB, Pinto JC, Monteiro JL et al., ESTIMA: "Um Pacote Computacional para Estimação de Parâmetros e Projeto de Experimentos”, Relatório Técnico PEQ/COPPE, Universidade Federal do Rio de Janeiro, Rio de Janeiro, RJ, Brasil, 1993.

15. Enzweiler H, Visioli LJ, Muneron de Mello JM, Guelli Ulson de Souza SMdA, Ulson de Souza AnA, Silva Ad, et al. Statistical Evaluation of Biochemical Kinetic Models for BTX Degradation. Ind Eng Chem Res. 2014 Dec 17;53(50):19416-25. doi: 10.1021/ie503408g.

16. Barros, VP. Escalas e simplificações - exemplos em sistemas físicos e biológicos. Rev Bras Ens Fís., pp.1-10. 2010. 